\title{
THE BRAUER GROUP OF A COMPACT HAUSDORFF SPACE AND $n$-HOMOGENEOUS $C^{*}$-ALGEBRAS ${ }^{1}$
}

\author{
ROGER HOWE
}

\begin{abstract}
The structure of all $n$-homogeneous $C^{*}$-algebras with a given compact Hausdorff space $X$ as maximal ideal space is looked at from a cohomological standpoint. Such algebras are matrix algebra bundles over $X$, and by means of fibrewise tensor products, a group, $B(X)$, analogous to the Brauer group of field theory, is constructed. A partial cohomological description of this group is given. Projective representations of finite groups are used to provide examples where $B(X)$ is precisely computable and nontrivial.
\end{abstract}

A $C^{*}$-algebra $A$ is called homogeneous of degree $n$ (or $n$-homogeneous) if all of its irreducible representations are of degree $n$. Though such algebras do not occur often "in nature", they are of some theoretical interest since they are the simplest noncommutative $C^{*}$-algebras, and indicate the kinds of phenomena which one may expect to occur for more general algebras (particularly if one considers their subalgebras) while remaining free of any taint of the various pathologies of the general case. They also have some aesthetic appeal.

The basic structure theorem for $n$-homogeneous $C^{*}$-algebras was established by J. M. G. Fell [3], and says:

Theorem. Let $A$ be an n-homogeneous $C^{*}$-algebra. Then the primitive ideal space $X$ of $A$ is a locally compact Hausdorff space, and if $A$ has an identity, then $X$ is compact. Moreover $A$ is naturally *-isomorphic to the algebra of continuous sections of an $n \times n$ matrix-algebra bundle over $X$.

By an $n \times n$ matrix-algebra bundle, we understand a vector bundle of dimension $n^{2}$, such that the fibres also have the structure of the algebra $M_{n}(C)$ (with the usual $C^{*}$ norm). We will be more technical in a minute.

The above theorem is a natural generalization of the theorem of GelfandNaimark [2], which asserts that a commutative $C^{*}$-algebra is * isomorphic

Received by the editors June 9, 1971.

AMS 1970 subject classifications. Primary 46L05; Secondary 22D25, 55F50.

Key words and phrases. $n$-homogeneous $C^{*}$-algebra, Brauer group, compact Hausdorff space.

${ }^{1}$ This work was supported by NSF Grant No. GP 19587.

(c) American Mathematical Society 1972 
to the continuous functions on its (Hausdorff, locally compact) maximal ideal space. Continuous functions may be regarded as sections of the trivial line bundle. Analogously, we have, for any locally compact Hausdorff space $X$, the algebra of continuous $M_{n}(C)$-valued functions, and this is an $n$-homogeneous $C^{*}$-algebra. Now, however, it is only one of several possibilities, for in Fell's theorem, nontrivial bundles definitely occur. Thus one may ask, for a given locally compact Hausdorff space $X$, what are the $n$-homogeneous $C^{*}$-algebras having $X$ as maximal ideal space? This question is not considered in detail by Fell, and, indeed, it leads into very complicated questions in nonabelian cohomology, so that one may despair of an answer in general. However, one may take a different point of view, provided by algebraic topology, and proceed to stabilize, homologize, linearize, and generally vastly simplify the problem, and still obtain some interesting information. It is this procedure we will expose in this note. It leads in particular to a natural analogue of the Brauer group of a field (or ring) for a compact Hausdorff space, and it has some connections with group representations. We also remark that the considerations here are closely related to those in [1], although the flavor is considerably different.

From now on, we will take $X$ compact. An $M_{n}(C)$-bundle $E$ over $X$ is a locally trivial fibre bundle over $X$, whose fibres have the structure of $M_{n}(C)$. Thus if $p: E \rightarrow X$ is the projection map, then we may find open subsets $U_{i} \subseteq X, i=1, \cdots, n$, such that $X=\bigcup_{i=1}^{n} U_{i}$, and such that there are fibrepreserving maps $d_{i}: p^{-1}\left(U_{i}\right) \rightarrow U_{i} \times M_{n}(C)$, such that $d_{j} \cdot d_{i}^{-1}:\left(U_{i} \cap U_{j}\right) \times$ $M_{n}(C) \rightarrow\left(U_{i} \cap U_{j}\right) \times M_{n}(C)$ is of the form $(x, T) \rightarrow\left(x, A_{i j}(x)(T)\right)$, where $A_{i j}(x)$ is an automorphism of $M_{n}(C)$. The $A_{i j}$ 's are called transition functions and satisfy the (noncommutative) cocycle relation $A_{j k} A_{i j}=A_{i k}$ and $A_{i j}=A_{j i}^{-1}$. We recall that to obtain an arbitrary vector bundle over $X$, you replace $M_{n}(C)$ by $C^{m}$ ( $m$ arbitrary) and let $A_{i j}$ be any linear isomorphism of $C^{m}$.

Now we consider the collection of all (isomorphism classes of) matrix bundles of arbitrary degree over $X$. Call this $M(X) . M(X)$ has a natural commutative semigroup structure, the multiplication being fibrewise tensor product. In terms of the local trivializations above, if $E_{1}$ is an $M_{n}(C)$ bundle, with transition functions $A_{i j}$, and $E_{2}$ is an $M_{l}(C)$ bundle with transition functions $B_{i j}$, then $E_{1} \otimes E_{2}$ is an $M_{n l}(C) \simeq M_{n}(C) \otimes M_{l}(C)$ bundle, with transition functions $A_{i j} \otimes B_{i j}$.

Now there is a distinguished subsemigroup $S M(X)$ of $M(X)$, consisting of matrix bundles of the form $V \otimes V^{*}$, where $V$ is an arbitrary complex vector bundle. That is, the isomorphism $W \otimes W^{*} \simeq \operatorname{Hom}(W, W)$ is well known for any vector space $W$, and if the transition functions for $V$ are $T_{i j}$, the transition functions for $V^{*}$ are $T_{i j}^{*-1}$, where ${ }^{*}$ denotes adjoints. 
Thus the transition functions of $V \otimes V^{*}$ are $T_{i j} \otimes T_{i j}^{*-1}$, which are automorphisms for the algebra structures on the overlapping trivializations.

Proposirion 1. The quotient $M(X) / S M(X)$ is a group. If $E \in M(X)$, then left multiplication of $E$ on itself defines an embedding $i: E \rightarrow \operatorname{Hom}(E, E)=$ $E \otimes E^{*}$. Then the commutant of $i(E)$ in $E \otimes E^{*}$ is $E^{*}$, the opposed algebra, which is $E$ acting on itself by right multiplication. $E^{*}$ is then an inverse for $E$ in $M(X) / S M(X)$.

The proof is already contained in the statement. In view of the evident analogy between $M(X)$ and central simple algebras (over a field, or their analogues over a ring), and between $S M(X)$ and the split algebras (this latter being particularly strong since the rise of algebraic $K$-theory), it seems reasonable to write $M(X) / S M(X)=B(X)$, and to call this the Brauer group of $X$. It is a useful object since it gives you a hold on the more "mysterious" part of $M(X)$. Presumably $S M(X)$ can be calculated, given a sufficient knowledge of $K(X)$.

As a corollary to Proposition 1, we note:

COROLlaRY. Every $E \in M(X)$ may be embedded in an algebra of matrix valued functions on $X$ (identities are not necessarily preserved).

Proof. Proposition 1 shows $E$ may be embedded in the $\operatorname{Hom}(V, V)$ for some vector bundle $V$ over $X$. Now for some large $n$, we may write $C^{n}=V \oplus W$, where $W$ is another vector bundle, and $C^{n}$ denotes the trivial $n$-dimensional bundle. Then we may embed $\operatorname{Hom}(V, V)$ in $\operatorname{Hom}\left(C^{n}, C^{n}\right)$ by extending $T \in \operatorname{Hom}(V, V)$ to be zero on $W$.

The next proposition provides a partial cohomological description of $B(X)$, the analogue of the situation for fields. The argument is essentially a direct limit over the low-dimensional cohomology exact sequence corresponding to $1 \rightarrow T \rightarrow U_{n} \rightarrow P U_{n} \rightarrow 1$, where $T$ is the circle, $U_{n}$ the $n$-dimensional unitary group and $P U_{n}$ the projective unitary group. The arguments are all quite standard, and are particularly closely related to [1].

We let $\mathscr{R}$ be the group of roots of unity in $C$. $\mathscr{R}$ has the discrete topology. We have the natural injection $\mathscr{R} \rightarrow T$. Given a group $G, H^{i}(X ; G)$ will denote the Cech cohomology of the sheaf of continuous functions of $X$ into $G$. Then if $G$ is discrete this is the usual Cech cohomology.

Proposition 2. There is a natural injection $d: B(X) \rightarrow H^{2}(X ; T) . d(B(X))$ is contained in the image of $H^{2}(X ; \mathscr{R})$ in $H^{2}(X ; T)$. In particular $B(X)$ is a torsion group. More specifically, if $E \in M(X)$ is an $M_{n}(C)$-bundle, then the order of $E$ in $B(X)$ divides $n$.

Proof. Let $E \in M(X)$, and let $\left\{U_{j}\right\}$ be an open covering of $X$, over each member of which $E$ is trivial. Let $A_{i j}$ be the transition functions. $A_{i j}(x)$ is an automorphism of $M_{n}(C)$, and all automorphisms of $M_{n}(C)$ are inner. 
Thus there is, for each $x, C_{i j}(x) \in M_{n}(C)$, such that $A_{i j}(x)(T)=$ $C_{i j}(x) T C_{i j}(x)^{-1}, T \in M_{n}(C) . C_{i j}(x)$ is determined up to constant. If we assume, as we may, that our $E$ actually is a $C^{*}$-algebra, then $A_{i j}(x)$ will be a *-automorphism, and $C_{i j}(x)$ may be taken to be unitary. If the $U_{i}$ 's are contractible, or in any case, by shrinking them if necessary, we may assume we have continuous functions $C_{i j}: U_{i} \cap U_{j} \rightarrow U_{n}$, such that conjugation by $C_{i j}$ gives $A_{i j}$, as above. Since $A_{j k} A_{i j}=A_{i k}$, the $C_{i j}$ 's satisfy the relation $C_{j k} C_{i j}=\alpha_{i j k} C_{i k}$, where $\alpha_{i j k}$ is a constant. Associating the product $C_{k l} C_{j k} C_{i j}$ in two different ways yields the identity $\alpha_{i j k} \alpha_{i k l}=\alpha_{j k l} \alpha_{i j l}$, so that $\alpha_{i j k}$ is a Cech 2-cocycle in the sheaf of $T$-valued functions. Now $A_{i j}$ is defined up to a coboundary, that is, we may replace $A_{i j}$ with $A_{i j}^{\prime}=B_{j} A_{i j} B_{i}^{-1}$, where $B_{i}: U_{i} \rightarrow \operatorname{Aut}\left(M_{n}(C)\right)$ is a continuous function. Similarly $C_{i j}$ may be replaced by $\beta_{i j} C_{i j}$, where $\beta_{i j}: U_{i} \cap U_{j} \rightarrow T$ is continuous. Either change only alters $\alpha_{i j k}$ by a coboundary (e.g., functions of the form $\beta_{i k}^{-1} \beta_{j k} \beta_{i j}$ ). Thus, we may associate to $E$ a well defined cohomology class $[\alpha]$ in $H^{2}(X ; T)$. Moreover it is clear that $[\alpha]$ is precisely the obstruction to splitting $E$. For, if $[\alpha]$ vanishes, we may alter $\alpha_{i j k}$ by a coboundary, to make $\alpha_{i j k} \equiv 1$. Then the transition functions $C_{i j}$ define an $n$-dimensional vector bundle $V$, and clearly $E \simeq V \otimes V^{*}$. On the other hand if $E$ splits, so that $E \simeq V \otimes V^{*}$, then taking the transition functions of $E$ to be those derived from $V$, we get the trivial cocycle, $\alpha_{i j k} \equiv 1$. Finally we observe that, according to the construction of tensor products using transition functions, we may construct a cocycle for $E_{1} \otimes E_{2}$ equal to the product of cocycles from $E_{1}$ and $E_{2}$. Thus our map $d: B(X) \rightarrow H^{2}(X ; T)$, which we have shown to be injective, is also a group homomorphism.

To prove the rest, we observe that, as $C_{i j}(x)$ is determined only up to a constant, then we may, by shrinking the $U_{i}$ 's if necessary, suppose that $C_{i j}(x) \in S U_{n}$, the special unitary group. Then $\alpha_{i j k} \in S U_{n}$, must be an $n$th root of unity. It is therefore locally constant. Cutting things further if necessary, we see $\alpha_{i j k}$ is in fact a Cech cocycle with values in the $n$th roots of unity, and a fortiori in $\mathscr{R}$. In particular, the class represented by $\alpha_{i j k}$ has order dividing $n$. This finishes Proposition 2.

We add some complements to Proposition 2. Following [1], we observe that the exact sequence $0 \rightarrow Z \rightarrow R \rightarrow T \rightarrow 0$ yields the cohomology sequence $H^{i}(X ; \boldsymbol{R}) \rightarrow H^{i}(X ; T) \rightarrow H^{i+1}(X ; Z) \rightarrow H^{i+1}(X ; \boldsymbol{R})$ which gives $H^{i}(X ; T) \simeq$ $H^{i+1}(X ; Z)$ for $i \geqq 1$, since $H^{i}(X ; \boldsymbol{R})=0$ for $i \geqq 1$, since the sheaf of continuous real-valued functions is "soft". Thus, in particular $H^{2}(X ; T) \simeq$ $H^{3}(X ; Z)$, the latter being an ordinary Cech group. Thus

COROllaRY 1. $B(X)$ is isomorphic to a torsion subgroup of $H^{3}(X ; Z)$. In particular, if $H^{3}(X ; Z)=0$ (or is torsion free) then every matrix bundle over $X$ is split. (Example: $T^{n}$.) 
Next, let $\mathscr{R}_{n} \subset \mathscr{R}$ be the $n$th roots of unity, and consider $0 \rightarrow \mathscr{R}_{n} \stackrel{i}{\rightarrow} T \stackrel{n}{\rightarrow}$ $T \rightarrow 0$ and the dual sequence $0 \rightarrow Z \stackrel{n}{\rightarrow} Z \rightarrow \mathscr{R}_{n} \rightarrow 0$. The first yields $H^{i}(X ; T) \stackrel{n}{\rightarrow}$ $H^{i}(X ; T) \rightarrow H^{i+1}\left(X ; \mathscr{R}_{n}\right) \stackrel{i}{\rightarrow} H^{i+1}(X ; T)$. The dual sequence results from the isomorphism $H^{i}(X ; T) \simeq H^{i+1}(X ; Z)$. This sequence gives

Corollary 2. If $H^{1}(X ; T) \simeq H^{2}(X ; Z)=0$ (or if it is divisible) then $i^{*}$ : $H^{2}\left(X ; \mathscr{R}_{n}\right) \rightarrow H^{2}(X ; T)$ is injective. Hence $H^{2}(X ; \mathscr{R}) \rightarrow H^{2}(X ; T)$ is injective, and $B(X)$ may be regarded as a subgroup of $H^{2}(X ; \mathscr{R})$.

Proof. We have the factorization $\mathscr{R}_{n} \rightarrow \mathscr{R} \rightarrow T$. If $i^{*}: H^{2}\left(X ; \mathscr{R}_{n}\right) \rightarrow$ $H^{2}(X ; T)$ is injective, so is $i^{*}: H^{2}\left(X ; \mathscr{R}_{n}\right) \rightarrow H^{2}(X ; \mathscr{R})$. Now $H^{2}(X ; \mathscr{R})$ is the direct limit of the $H^{2}\left(X ; \mathscr{R}_{2}\right)$ 's, and $i^{*}: H^{2}(X ; \mathscr{R}) \rightarrow H^{2}(X ; T)$ is the corresponding direct limit of maps. If $H^{2}\left(X ; \mathscr{R}_{n}\right) \rightarrow H^{2}(X ; \mathscr{R})$ is injective, then $H^{2}(X ; \mathscr{R})$ is just the union of the $H^{2}\left(X ; \mathscr{R}_{n}\right)$. Since each of these is mapped injectively into $H^{2}(X ; T)$, so is $H^{2}(X ; \mathscr{R})$.

In general, since $\mathscr{R} \simeq Q \mid Z$, we may compute the kernel of $i^{*}: H^{2}(X ; \mathscr{R}) \rightarrow$ $H^{2}(X ; T)$ by the sequence $0 \rightarrow Z \stackrel{i}{\rightarrow} Q \stackrel{\rho}{\rightarrow} Q / Z \rightarrow 0$, which gives $H^{2}(X ; Z) \stackrel{i}{\rightarrow}$ $H^{2}(X ; Q) \stackrel{\rho}{\rightarrow} H^{2}(X ; Q / Z) \rightarrow H^{3}(X ; Z)$. The universal coefficient theorem gives $H^{2}(X ; Q) \simeq H^{2}(X ; Z) \otimes Q$. We may thus recover Corollary 2 , and specify the kernel more exactly. We will explicitly mention the opposite extreme from Corollary 2.

COROLlaRY 3. If $H^{3}(X ; Z)$ is finitely generated, then $B(X)$ is a subgroup of the quotient $H^{2}(X ; Q / Z) / D H^{2}(X ; Q / Z)$, where $D H^{2}$ means the subgroup of $H^{2}$ consisting of divisible elements. In particular, $B(X)$ is finite.

Proof. If $H^{3}(X ; Z)$ is finitely generated, it contains no nontrivial divisible subgroups. Hence $D H^{2}(X ; Q / Z)$ is certainly in the kernel of $i^{*}$ on $H^{2}(X ; Q / Z)$, since the image of a divisible group is divisible. On the other hand, $H^{2}(X ; Q)$ is divisible always, and so for the same reason, $\rho\left(H^{2}(X ; Q / Z)\right) \subseteq D H^{2}(X ; Q / Z)$. Then $B(X)$ is a torsion subgroup of a finitely generated group, hence finite.

COROllary 4. If $X$ is a finite simplicial complex, then $B(X)$ is a finite group.

Proof. $H^{i}(X ; Z)$ is then a finitely generated group.

There is an interesting class of examples of the foregoing where one can be more precise. Suppose $X$ is a finite simplicial complex, $G$ a finitely generated discrete group and $Y$ a principal $G$-bundle over $X$. We assume that $\pi_{i}(Y)=0$ for $i \leqq 3$. Then $Y$ is the universal cover of $X$ and $\pi_{1}(X) \simeq G$. In particular, if $G$ has finite cohomological dimension, $Y$ may be contractible and $X$ a $K \pi_{1}$ space.

By virtue of our assumptions $H^{i}(Y ; Z)=0$ for $i \leqq 3$, and $H^{i}(X ; Z)$ and $H^{i}(G ; Z)$ are naturally isomorphic ( $Z$ being a trivial $G$ module) for $i \leqq 3$. 
(This is essentially folklore.) Similar statements hold for coefficients in $Q$ or $Q / Z(=\mathscr{R})$. Suppose $\rho: G \rightarrow P S U_{n}$ is a projective representation of $G$. Then we can form $E=\left(M_{n}(C) \times Y\right) / G$, in the usual way, and this will be a matrix algebra bundle over $X$. Now the obstruction to lifting $\rho$ to a bonafide representation $\tilde{\rho}: G \rightarrow U_{n}$ is an element of $H^{2}(G ; \mathscr{R})$, the so-called Mackey obstruction. It is clear from the construction of the isomorphism between $H^{i}(G ;)$ and $H^{i}(X ;)$ for $i \leqq 3$ that the corresponding class in $H^{2}(X ; T)$ is $d(E)$. Thus one may calculate that $B(X)$ in this case is a subgroup of $H^{2}(G ; \mathscr{R}) / D H^{2}(G ; \mathscr{R})$; namely the image of the group generated by the Mackey obstructions.

Suppose now $G$ is finite. Then $D H^{2}(G ; \mathscr{R})=0$, and every element of $H^{2}(G ; \mathscr{R})$ is realizable as a Mackey obstruction. For, given $\alpha \in H^{2}(G ; \mathscr{R})$, one may construct the corresponding central extension of $G$. Then corresponding to the identity character on the center, one may find an irreducible finite dimensional representation of the extended group. This then defines a projective representation of $G$, with Mackey obstruction $\alpha$. We state this as our final result.

Proposition 3. If $\rho: Y \rightarrow X$ is a principal $G$ bundle, for $G$ finite, $X, Y$ finite complexes, and $\pi_{i}(Y)=0$ for $i \leqq 3$, then $B(X) \simeq H^{2}(G ; \mathscr{R})$.

In particular this provides examples of nonsplit matrix bundles, and is probably the situation which most approaches the classical case of fields.

\section{REFERENCES}

1. J. Dixmier and A. Douady, Champs continus d'espaces hilbertiens et de $C^{*}$-algèbres, Bull. Soc. Math. France 91 (1963), 227-284. MR 29 \#485.

2. J. Dixmier, Les $C^{*}$-algèbres et leurs représentations, Cahiers Scientifiques, fasc. XXIX, Gauthier-Villars, Paris, 1964. MR 30 \#1404.

3. J. M. G. Fell, The structure of algebras of operator fields, Acta Math. 106 (1961), 233-280. MR 29 \#1547.

Department of Mathematics, State University of New York at Stony Brook, StONY BROOK, LONG IsLAND, NeW YORK 11790 